\title{
Moral Education from the Perspective of Moral Belief
}

\author{
$\mathrm{Yu} \mathrm{Li}^{1} \&$ Lianyong Xiong ${ }^{2}$ \\ ${ }^{1}$ The Student Work Department, Sichuan Agricultural University, Chengdu, China \\ ${ }^{2}$ College of Landscape Architecture, Sichuan Agricultural University, Chengdu, China \\ Correspondence: Yu Li, The Student Work Department, Sichuan Agricultural University, Chengdu 611130, \\ Sichuan, China. E-mail: liyuxtw@163.com
}

Received: December 4, 2012 Accepted: January 8, 2013 Online Published: February 28, 2013

doi:10.5539/ass.v9n3p107 URL: http://dx.doi.org/10.5539/ass.v9n3p107

\begin{abstract}
This paper analyzes reasons for low efficiency in moral education in ideological and political education in China at present and comes up with the idea of promoting the efficiency of moral education from the following three aspects of moral belief education, construction of core moral value system and cultivation of moral emotion.
\end{abstract}

Keywords: moral belief, moral education

The efficiency in moral education in China is low and when we explore the reasons underlying, we find that avoidance from the core value and ultimate value of morality in moral education is an important reason. Either in school or in social education, the moral education purely gives a brain-wash style of preach to students superficially, but ignores cultivation of their moral belief, whereas effective moral education is supposed to attach great importance to cultivation of moral belief.

\section{Implication and Characteristics of Moral Belief}

"Belief is the extreme conviction and worship of people on a certain kind of proposal, principle or value ideal and it embraces people's highest spiritual care." However, moral belief is a kind of special belief and is a kind of belief in and worship on a certain kind of moral value and principle by people who earnestly practice what they advocate. It is the reflection of the highest pattern of moral development and it has the following important characteristics.

\subsection{Moral Belief Is the Footstone of People's Moral Behavior}

Belief is classified, from different perspectives, into several types. In terms of content, it can be classified into political belief, moral belief and legal belief and so on. Formation of moral belief originates from habit and is usually hard to be formed within a short period of time. Yet, once moral belief is formed, it will provide everlasting and stable spiritual motive for people's choice of moral behavior and become a stable and strong moral personality. Moral belief is conviction and worship of people on morality and moral belief is based on the standard of moral principle and moral norm and on the behavioral motive and the result generated. It makes judgment on its own behavior and adjusts its behavior. Thus, essentially, it is the self-transcendence of its moral personality and it imposes pressure on people's spirit and makes the concept become the major standard for our behaviors. Cultivation of moral belief helps to strengthen social stability and harmony. Therefore, moral belief is the footstone of people's moral behavior and is the starting point for people to judge whether their behaviors are moral. Sometimes, it has no absolute rational evidence in accordance with the emotion.

It is analyzed from the perspective of psychology that quite a lot of personality disturbance and moral degeneration is closely connected with loss and indifference of moral belief. During the period of social transition when diversified cultural conflicts and integration exist, the value concept is at a state of contradiction and perplexity which has quite direct and unfavorable effects upon the psychological health of people. And these unfavorable effects are manifested as anti-social personality, borderline personality, temporization type of personality and compliance personality.

\subsection{Moral Belief Functions by Means of Moral Value System}

Moral belief is indispensable in the entire belief system. The well-known German classical philosopher Kant made most statements on moral belief and gave a high evaluation on moral belief. In the philosophical system of Kant, essentially speaking, belief referred to moral belief and had an extremely important status. To a certain 
extent, it can be said that the ethics philosophy of Kant is a kind of moral belief theory. Kant classified belief into practical belief, doctrinal belief (or dogmatic belief and dogmatical belief) and moral belief, and believed that practical belief was accidental and was closely connected with the actual strategy of a certain behavior. The doctrinal belief means that a judgment in theory merely attains the degree of subjective confirmation, but has no sufficient evidence. Moral belief is the highest level of belief, the conviction and obedience of moral law and "absolute command", and is unconditional and absolute. Moral belief is conviction and obedience of moral law or absolute command that breaks away all interferences and is a kind of unconditional and absolute moral belief with determination, stability and absolute necessity.

Nevertheless, moral belief plays its specific constraining role in people's behavior by means of specific moral value system. When people conduct themselves specifically, it is not possible to merely depend on such highly abstract moral principles as "no self-interest at all and specially for others" or "one for all and all for one" as a reference. Instead, it is a complete value system and specific standard established on the basis of such principle that is regarded as a reference for people's behaviors.

\subsection{Moral Belief Is Supported by Moral Emotion}

Moral belief is established on the basis of people's rational cognition, but support of moral emotion is necessary in order to firmly believe in a certain kind of good principle and value. To a certain extent, moral belief means "irrational expression based on rational identity". "Moral belief is the highest existence of moral emotion, and when the moral emotion is developed to the extent of doubtless and firm believing, passionately devoting, being as fervor as the fire and as concentrated as the mountain, it developed into moral belief.

Moral belief is supported by emotion and only people with deep moral emotion have firm moral belief and will spontaneously perform their moral belief and standard. Their external presentation is that they are able to perform any moral responsibility and obligation consciously and on their own initiative according to a moral norm and requirement. There is no need to impose any external constraint on them. And when they fail to do so, they will have a feeling of abashment, compunction and self-reproach. On the contrary, a person with flattening and numbness of moral emotion is indifferent to the moral responsibility and obligation. And even if they behave according to the moral norm and requirement under the compelling of an external force, it is contrary to his conviction, which is usually manifested as two-faced characteristics. In addition, moral belief also generates moral emotion. Loss and moral belief will necessarily lead to indifference and numbness of moral emotion.

Furthermore, to a certain extent, moral emotion originates from the habit. According to David Hume, "The unique origin for all beliefs is habit", and only the principle of habit beneficially constructed our experiences and made us expect that a succession of events would occur in the future that were similar to those in the past. Without influences of the habit, we had no way to predict all those facts beyond the so-called direct and immediate facts so far as our memory and sense organ were concerned. In this sense, without doubt, belief is a kind of necessary consciousness that is evoked by a connection principle. Hence, as a sort of belief, formation of moral belief can only originate from the habit.

\section{Prominent Problems in Moral Education in China from the Perspective of Moral Belief}

\subsection{Loss of Cultivation of Moral Belief}

At present, the moral education mode in China mainly preaches superficially and tells what one should do and what one shouldn't do, lacking in cultivation of moral belief at a deep level. Under such a kind of moral education mode, the deep level of moral standard lacks its support and the moral standard almost becomes a rootless duckweed, so it is difficult to enjoy popular support and when people are faced up with choice of moral behaviors, they tend to wave with the wind. Besides, for the time being, there exists a wrong kind of "utilitarianism" moral value concept. That is to say, under the circumstance when cultivation of moral belief is lacking, people summarize the reasons for moral demand as that the behaviorists will get long term interest return, which, as a matter of fact, has an extremely great negative effect upon moral education.

To a certain extent, deficiency of cultivation of moral belief may originate from the fact that quite a large part of people in China hold a biased attitude toward the belief for a long time and that they understand the belief, without consciousness, as religious belief or political belief. And it seems not to have the concept of moral belief in their mind. As for religious belief, on the basis of adhering to the basic viewpoint of materialism, we make a profound criticism on its basic world outlook, which leads to the result that the moral value outlook advocated by religious belief is not convinced by a large part of people at the present stage in China. The mainstream Marxism belief in China is more a kind of political belief. Although it is scientific and has become a dominant ideology in terms of politics, it is difficult to play a constraining role in the moral behaviors in the daily life. Therefore, 
actually, the moral belief is still at a state of deficiency.

\subsection{The Core Value System of Morality Is in Chaos}

As the dominant political belief, Marxism also provides some basic world outlook guide and methodology for other beliefs. However, in the Marxism belief system, a distinct and complete core value system has not been constructed in terms of moral belief. In such a kind of incomplete and ambiguous value system, moral belief is incapable of playing an effective role and is unable to provide specific behavioral guidance for the moral behaviors of people in their life, which leads to chaos of moral belief in the society where some people "partly" refer to the religion, some "partly" refer to the traditional ethics and some refer to the westernized moral belief. Under the circumstance of diversification, the mainstream socialist moral value belief system has not been constructed.

The inanition of moral value system in China, on one hand, reveals that a complete system has not been constructed in terms of theory and even the basic principles advocated by the mainstream media are contradictory. On the other hand, the inanition also reveals the fact that the recognized value still has not obtained sufficient publicity and propaganda strength and the entire society lacks sufficient recognition in the mainstream core value of moral belief, which leads to the result that the influence of the mainstream value is fragile and has not occupied a due position of the mainstream value. When we explore the reasons underlying, we find that doctrinal application of Marxism might be one of the important reasons, which lacks improvement and innovation of Marxism belief in moral theory. In terms of political theory, we have made epoch-making development and innovation of Marxism and we obtain such theoretical achievements as Mao Zedong Thought and Deng Xiaoping Theory that both combined the reality of China. However, by contrast, in terms of moral belief theory, up to now, there has not had any great theoretical achievement.

\subsection{Cultivation of Moral Emotion Is Lacking}

Moral belief is supported by moral emotion and is a kind of belief with "irrational expression based on rational identity". Formation and influential strength of moral belief, to a large extent, rests with emotion of the society and individuals to morality. Religious belief usually resorts to a variety of solemn church services imbued with culture and contagious fairy stories to consolidate this kind of emotion. Yet, in our ideological and political education, morality almost purely becomes a kind of preaching education in which the inculcating practice and boring language in the rigid education concept and educational process leads to the result that the young people who accept this kind of educational mode always keep to some doctrines in Marxism and that some people have the feeling of antipathy and rejection whose moral belief lacks emotional appeal of the soul. Therefore, people show indifference to the moral standard emotion they advocate and weaken the power of morality, which makes their cognition and behavior separated. That is to say, even if they are aware what is wrong, they still don't hesitate to do it.

Indifference of moral emotion might result in numbness and indifference of the public when people's morality suffers from damage and cause the bottom line of morality to be often challenged, which, maybe, is one of the reasons why people are numb to all kinds of events that publicly violate morality. In the meantime, weakness of moral emotion may get people to experience painful suffering of the soul when they are faced up with a tempt and even may fail to resist the trial of the tempt. In the face of tempt of a benefit, those with firm moral belief and fervor moral emotion will not suffer from pain of the soul. On the contrary, people might vacillate when making a choice. They have to suffer from a feeling of loss and pain when they vacillate between selfishly choosing "self-interest" or morally choosing "benefiting others". The degree of firmness of moral belief is closely connected with the subjective happiness index of people in the reality.

The above might be a unique problem of morality in China. On one hand, Marxism is supposed to stand its ground with its scientific and rational belief features, whereas it is not recognized and accepted. On the other hand, the individuals set up, one after another, the so-called extremely bad, degenerated and blurry personal belief in the extremely complicated society. People often drift away between worship of personal value and derogation of the collective value. Thus, they are indeterminate and at a loss end, which leads them to go towards disillusion of belief and perplexity of the life. Social belief and personal belief are seriously departed, and, meanwhile, the crisis emerges, as a result of which people add weight to their own crisis just because of the crisis of others.

\section{Approaches to Moral Education from the Perspective of Moral Belief}

\subsection{Cultivation of Moral Belief as a Footstone of Moral Education}

At present, in order to change the status quo of low efficiency in moral education and enable moral education to 
get its ideal effect, much emphasis should be placed on cultivation of people's moral belief in moral education instead of purely giving a preach about moral standard at a technical level. Conviction and obedience of moral belief to moral law and "absolute order" is unconditional and absolute. Only when a social subject has a moral belief, can the specific standard of morality have its support and footstone.

Belief is conviction and worship of people in a certain kind of theory, principle or doctrine who regard it as their own behavioral norm and activity guidance. It is the fundamental standard and attitude that determines what one should do and shouldn't do, a part of belief, and the most concentrated and highest manifestation means of belief. It is the choice of people for their outlook on life, values and world outlook. Yet, moral belief education refers to the process in which a certain society and social class provides guidance and offers education on the world outlook, outlook on life and values of people. Cultivation of moral belief needs to be conducted in the school and social education and also requires the joint participation of the whole society. Under the "human-oriented" education idea, conscious preaching and normalizing can expect to establish a stable belief in the depth of the soul of educatees and make education of moral standard have its own footstone and starting point.

\subsection{Construction and Publicity of a Complete Moral Core Value System}

It is necessary to pay attention to the following several aspects in the process of constructing the core moral value system. In the first place, in terms of content, we need to avoid abstract and empty value standard and materialize the belief concept of a basic value idea. After all, a basic value idea is relatively abstract, whereas re-construction of a belief calls for maneuverability in practice. This requires us to materialize the basic idea, make it close to the practical activities of people in the daily life and set forth to construct the specific content of socialist core value system, such as, socialist Eight Honors and Eight Shames, social public morality, professional ethics and family virtues, etc. Only a materialized moral standard can penetrate into every corner of people's life, enable people to really appreciate its existence and play its role in normalizing and constraining people's behaviors to a maximum extent. Instead, it will not have no reference at all under an empty slogan.

In the second place, setting of moral standard should avoid extremalization, and, instead, each level of setting should have a standard. Then, the moral standard can play a better role in constraining people's behaviors. Currently, the value standard we have set tends to have the characteristics of extremalization. Either it is lofty of "no self-interest at all and specially for others", or it is contemptible of "harming others to benefit oneself". There is almost no standard for reference at an intermediate zone. However, a large majority of people in the reality are at an intermediate morality state and either this or that standard actually makes a large majority of these people lose their standard, as a result of which they are in perplexity and which is hard to effectively constrain people's behaviors.

In the third place, it is necessary to deal with the relationship between Marxism mainstream and the traditional, westernized and religious ideas in a correct way. At the present time, the theoretical origin of our social moral belief is chaotic in that it not only contains Marxism elements, but also has traditional, westernized and religious ideas. As a dominant belief, Marxism ought to take a leading position in the domain of moral belief. However, its statement of the theoretical ideas in domain of moral belief is too abstract, which makes it difficult to form a complete moral core value system. In order to keep pace with the times, it is necessary for the construction of moral core value system to inherit the excellent elements in the traditional, westernized and religious ideas, which is indispensable to construct a complete system. Quite a lot of moral ideas in the tradition, in the western world and in the religion have had a certain root in their dissemination among our social public. Still, there exists the problem that dross and quintessence co-exist. Thus, it is a topic to be discussed how to absorb the quintessence at the time of abandoning the dross. On one hand, this requires discrimination of the entire academic world in terms of theory, as quite a lot of ideas are complicated, which means that quintessence exists in dross and dross hides in quintessence. On the other hand, the part of quintessence discriminated needs to be integrated in terms of theory so as to construct a complete system. Furthermore, in order for a kind of theoretical system to become a real mainstream consciousness in the mind of all the people in the whole society, vigorous publicity and promotion is required, that is to say, joint efforts of the whole society are required.

\subsection{Cultivation of Moral Emotion}

The former Soviet Union educator Sukhomlinsky ever said, "Emotion is the core and flesh and blood of moral belief, principle and spiritual power. Without emotion, morality will become cut-and-dried and empty talk, which can only foster hypocrites." Moral emotion is the support of moral belief and without firm conviction in moral value, moral belief wouldn't have been formed. Cultivation of moral belief among young people should not only lay emphasis on improvement of moral cognition, but should also consolidate exercise of moral will, formation of moral habit and edification of moral emotion and constantly solidify external moral norms into internal moral 
integrity. Just as religious belief, moral belief also has a transcendental feature and has emotional factors. Cultivation of moral belief has to be conducted on the basis of cultivation of moral emotion. When moral belief starts from such irrational perspective of emotion, it can get more recognized and accepted by the public, so as to find a realistic breakthrough for construction of socialist moral belief. In other words, re-construction of moral belief should not merely resort to abstract theoretical demonstration.

Hence, cultivation of moral emotion needs to attach great importance to scientificalness, innovation and artistic features of moral education method in the process of education. On one hand, it is necessary to create moral education activities with abundant spiritual life content in moral education, on the basis of which the public are able to personally appreciate the emotional edification and influences when they participate and they may then experience the standard of moral concept and moral norm with affection. On the other hand, it is also necessary, in the process of moral education, to emphasize experience of moral emotion and enable people to get their moral emotion purified and enhanced in the process of performing moral responsibility and obligation. This kind of emotional experience needs a corresponding environment and social atmosphere. Such irrational beliefs as religious belief have provided quite a lot of experiences and methods to learn from. The huge contrast between pseudoscience of religious doctrine and popularity speed of religion indicates that a simple and clear-cut elaboration mode is indispensable for establishment of belief.

In addition, education of moral belief needs to resort to popular and simple language and detailed humanization content to assist the vast number of public in their acceptance and approval. In the realistic life, moral education has to focus on the emotional life and spiritual contradiction of people, go back to "the worldly earth" from the "paradise", shift its mode of discourse, get rid of the abstract theoretical terms, gain understanding and approval of the public with a distinct, simple, understandable and unadorned language style and reasoning manner, enter into their realistic life, go deep into their inward world and find a home to return to for their daily behaviors.

\section{References}

Gao, Z. M. (2001). The Believing Crisis during the Social Transformation. Zhejiang Social Sciences, (1).

Hume, D. (1980). A Treatise of Human Nature. Translated by Guan, Wenyun. Beijing: The Commercial Press.

Li, R. (2010). Several Thoughts on Re-construction of Belief in Contemporary China. Exploration and Free Views, (6).

Ma, D. X., \& Wang, M. L. (2010). Moral Belief and Moral Education. The Modern Education Journal, (5).

Tan, C. B. (1999). Belief Education and Moral Education. Beijing: Education Science Publishing House.

Zhu, M. J. (2010, May 12). Emotion Is the Flesh and Blood of Morality. Retrieved from http://www.xjdaily.com 